\title{
Aplicación de la plataforma KAHOOT en asignaturas de Ingeniería de Fabricación
}

\section{M.A. Sellés ${ }^{a}$, S. Sánchez-Caballero ${ }^{b}$, Elena Pérez-Bernabeu ${ }^{c}$}

${ }^{a}$ Departamento de Ingeniería Mecánica y de Materiales. Campus de Alcoy. Universidad Politécnica de Valencia. E-mail: maselles@dimm.upv.es, 'Departamento de Ingeniería Mecánica y de Materiales. Campus de Alcoy. Universidad Politécnica de Valencia. E-mail: sasanca@dimm.upv.es, ${ }^{\mathrm{c} D}$ Departamento de Estadística e Investigación Operativa Aplicadas y Calidad. Campus de Alcoy. Universidad Politécnica de Valencia. E-mail: elenapb@eio.upv.es

\begin{abstract}
Several tools that support online teaching have been released in the last few years, leaving the Personal Response Systems as obsolete systems. Among them, KAHOOT is widely used in the world. In this study, two subjects of manufacturing are used to implement this platform during two academic years. The obtained results in terms of academic improvement and motivation of students make completely advisable to use this free platform in engineering classes.
\end{abstract}

Keywords: KAHOOT, gaming, manufacturing, engineering, motivation, academic improvement

\begin{abstract}
Resumen
En los últimos años han aparecido varias herramientas online de apoyo a la docencia, dejando a los Personal Response Systems como sistemas obsoletos. Entre ellas, KAHOOT es de la más utilizada a nivel mundial. En este trabajo se utilizan dos asignaturas de Ingeniería de Fabricación para poner en práctica esta plataforma durante dos cursos académicos. Los resultados obtenidos en cuanto a la mejora académica y de motivación de los alumnos hacen totalmente aconsejable el uso de esta plataforma gratuita en las clases de Ingeniería.
\end{abstract}

Palabras clave: KAHOOT, Gaming, Fabricación, Ingeniería, Motivación, Mejora Académica

\section{Introducción}

La mejora del aprendizaje de los estudiantes de Ingeniería es una de las principales preocupaciones que desde siempre han tenido las universidades. Desde siempre ha habido preocupación por parte de los docentes para conseguir que sus alumnos logren retener y comprender la mayor cantidad de conocimiento posible. 
Internet y la introducción de la multimedia en las aulas ha posibilitado que los alumnos puedan experimentar situaciones reales que complementan su formación de una forma mucho más visual. En este sentido se ha avanzado muchísimo, gracias en parte a los cursos y herramientas online.

Entre estas herramientas, está la posibilidad de realizar juegos en el aula. ¿A quién no le gusta jugar con sus compañeros? A este concepto se le conoce con el término anglosajón "gaming". Uno de los problemas actuales en la universidad es la falta de motivación en los alumnos, y con este tipo de juegos se estimula claramente la participación y la motivación.

A finales de los 90 y principios de este siglo, aparecen los Personal Response Systems (PRS), que son unos dispositivos formados por un programa específico que se instalaba en el ordenador del profesor, un receptor inalámbrico, y unos transmisores por infrarrojos que se entregaban a los alumnos para poder almacenar sus respuestas a preguntas tipo test. Cuando el profesor realizaba una pregunta, los estudiantes podían responder a través de estos transmisores. Todas las respuestas de los alumnos se guardaban y se analizaban, y podían además mostrarse a través del proyector de la clase en una pantalla. El sistema se podía programar también para que mostrase la identidad del alumno que hubiese respondido correctamente antes que cualquier compañero.

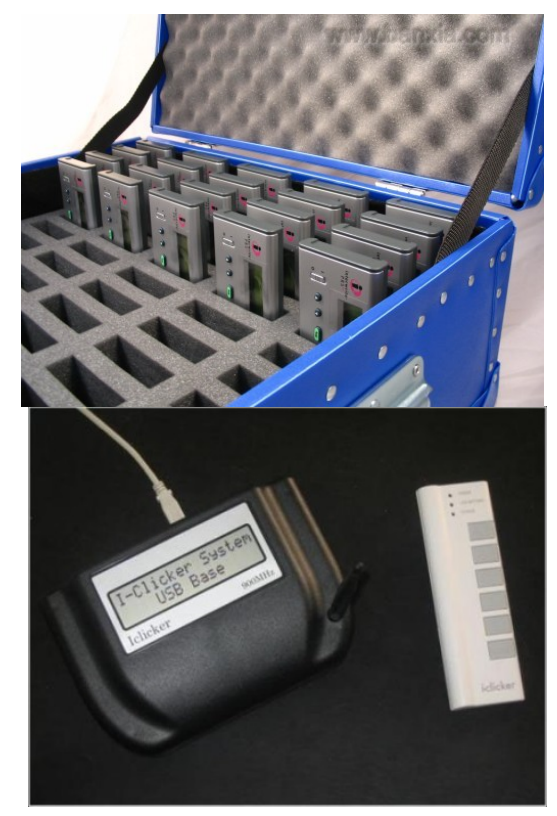

Fig. 1 Elementos que se utilizan en los PRS: mandos y base receptora de infrarrojos

Los PRS han quedado rezagados a un segundo plano por los dispositivos móviles de los que disponen la gran mayoría de los alumnos, conocidos como "smartphones". La facilidad de uso de estos dispositivos, más rapidez en el uso, así como su mantenimiento nulo por parte del profesor, y bajo coste para el mismo, ha propiciado que plataformas como KAHOOT, SOCRATIVE, PINNION, POLLEVERYWAY, GOOGLE FORMS Y QUESTIONPRESS, hayan tenido mucho éxito. 


\section{Estudios previos}

Son varios los estudios que recientemente se han llevado a cabo relacionados con el "gaming", y en particular, con la plataforma KAHOOT. Pintor y otros (Pintor, 2014) realizaron un estudio con el KAHOOT en una asignatura de odontología en la que se demostró que el uso de esta herramienta aumentó el grado de atención de los alumnos en clase, así como su mejora en cuanto a resultados académicos.

Del Cerro (Del Cerro, 2015) intenta paliar la falta de interés en su asignatura del Ciclo Formativo de Alojamientos Turísticos a través de la gamificación, planteando una serie de juegos y actividades distintos en cada unidad. Entre ellos, se utiliza KAHOOT. Los resultados del aprendizaje fueron positivos, ya que los alumnos alcanzaron los objetivos de aprendizaje de la asignatura y finalizaron el curso, con una visión más positiva del departamento de pisos o housekeeping, así como de la labor que realizan las diferentes categorías profesionales que lo conforman.

Hace unos meses se ha publicado un estudio de la universidad de Trinidad y Tobago (Perdue, 2016) sobre el uso de juegos en las clases de matemáticas. En él, se utiliza el portal CLASSCRAFT para convertir las clases en un juego de rol, donde los alumnos pueden ganar en nivel de experiencia. KAHOOT también se utiliza. Como conclusión, la autora pone de manifiesto la mejora en cuanto a atención en clase, en resultados académicos, y como novedad, la recompensa por los deberes bien hechos o las respuestas acertadas.

Thorine y Gårdsrud (Thorine, 2014) realizaron un estudio profundo de la plataforma KAHOOT. En él analizaron el retraso en la comunicación wifi, la posición en el aula del alumno y otras variables como el tiempo medio de respuesta.

\section{Objetivos}

Los objetivos que se pretenden con el presente estudio son:

- Aplicación de la plataforma KAHOOT en dos asignaturas de Ingeniería de Fabricación.

- Mejorar el rendimiento académico de los alumnos.

- Aumentar su motivación e interés en las clases.

- Llevar las asignaturas al día.

- Aumentar el grado de atención en clase.

Para ello, se han seleccionado dos asignaturas de cuarto curso de Ingeniería Mecánica (Campus de Alcoy de la Universidad Politécnica de Valencia), con un número de alumnos reducido: Fabricación Asistida por Ordenador y Sistemas Integrados, y Procesos de Conformado de Materiales Compuestos y Poliméricos.

(cc) EY-NC-ND 2016, Universitat Politècnica de València

Congreso In-Red (2016) 


\section{Desarrollo}

La plataforma KAHOOT se ha introducido en las clases de estas asignaturas en los cursos académicos 2014/2015 y 2015/2016. Son asignaturas semestrales, y los resultados académicos del curso 2013/2014 se han utilizado para realizar una comparativa con la plataforma.

El empleo de la plataforma se ha realizado en clase siempre al principio de cada sesión. De esta forma, con una duración inferior a 15 minutos, se planteaba un juego de 20 preguntas sobre el contenido explicado el día anterior. Los alumnos, muy motivados porque después de cada pregunta aparece una clasificación provisional, siempre han repasado los contenidos de cada tema antes de la sesión. La rivalidad entre ellos y la competitividad sana, ya que los alumnos querían aparecer entre los cinco primeros en la clasificación final de las asignaturas (puestos que obtenían como premio entre 0’5 y 0’1 puntos adicionales sobre la nota final de la asignatura, según la posición alcanzada), han hecho que KAHOOT fuese ya determinante en su quehacer diario.

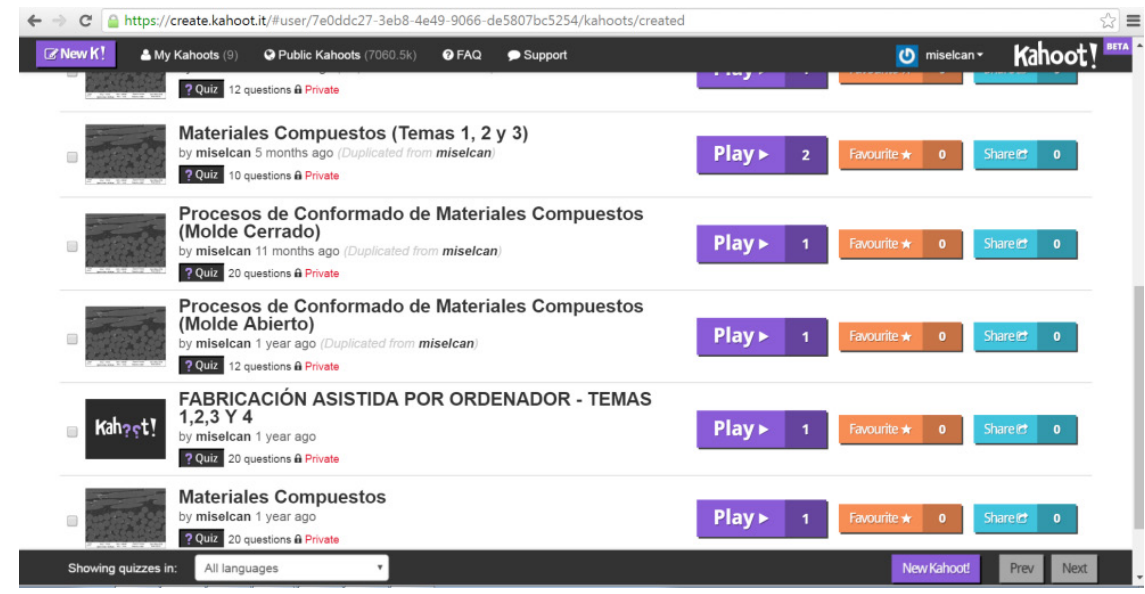

Fig. 2 Algunos de los KAHOOTS creados para las dos asignaturas

Las botellas de plástico se fabrican por...

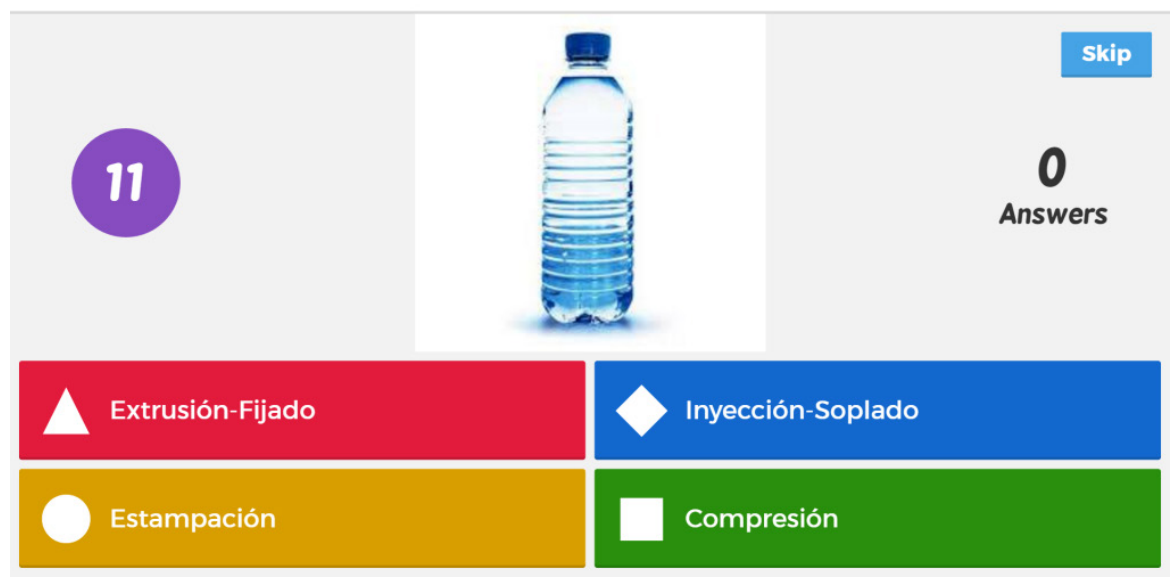

Fig. 3 Una pregunta en KAHOOT

(cc) EY-NC-ND 2016, Universitat Politècnica de València

Congreso IN-RED (2016) 
Otros profesores del Campus de Alcoy de la UPV han empezado a implementar KAHOOT en sus clases, con otras pequeñas variantes, como pueda ser por ejemplo la utilización de la plataforma al finalizar cada sesión de clase, para conocer si los alumnos han estado atentos o no. Se ha demostrado que si se les avisa que al finalizar la sesión van a participar en un concurso de preguntas sobre lo explicado, la atención de los alumnos aumenta más de un 70 $\%$.

\section{Resultados}

Como se ha comentado anteriormente, la plataforma KAHOOT se ha utilizado en clase durante los dos últimos cursos académicos.

Por lo que respecta a la asignatura Fabricación Asistida por Ordenador y Sistemas Integrados (FAOSI), la nota media de todos los alumnos en el curso 2013/2014 fue de 6,85 puntos. Desde que se utiliza KAHOOT, la nota media ha ascendido hasta 8,13 y 8,43 en los cursos 2014/2015 y 2015/2016, respectivamente.

En cuanto a la asignatura Procesos de Conformado de Materiales Compuestos y Poliméricos (Compuestos), la nota media de todos los alumnos en el curso 2013/2014 fue de 7,27 puntos. Desde que se utiliza KAHOOT, la nota media ha ascendido hasta 8,31 y 8,10 en los cursos 2014/2015 y 2015/2016, respectivamente.

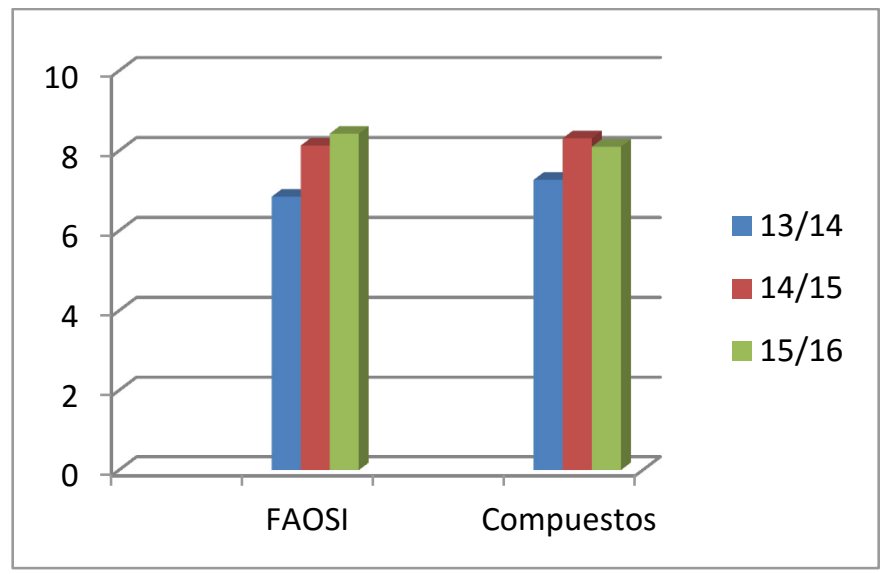

Fig. 4 Nota media de los alumnos en cada uno de los tres cursos académicos

El grado de satisfacción de los alumnos por el empleo de la plataforma queda plasmado en la Fig. 5, observándose que es muy alto. 


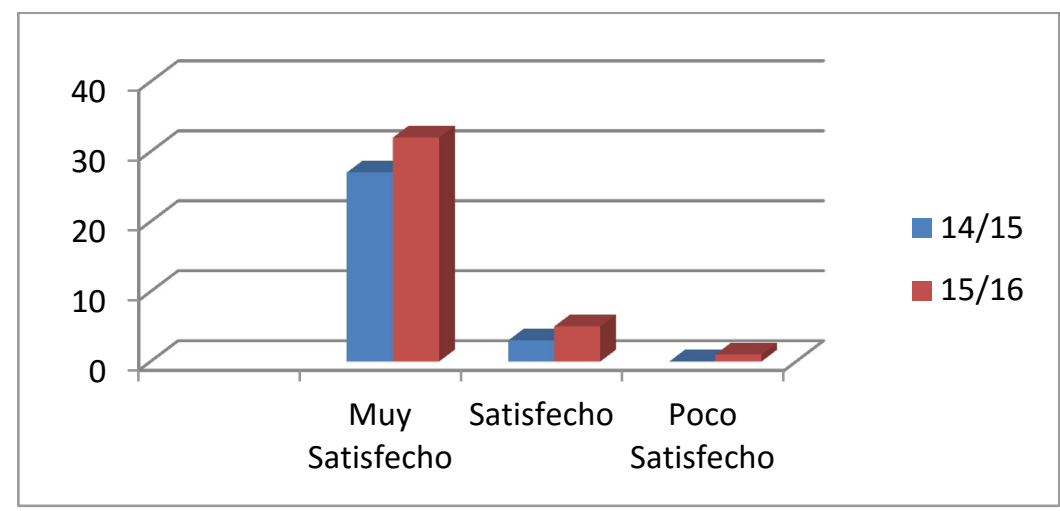

Fig. 5 Grado de satisfacción de los alumnos con el uso de KAHOOT en clase

\section{Conclusiones}

Se ha comprobado como la utilización de esta herramienta interactiva puede aumentar la participación de los estudiantes en clase, aumentando la atención durante la misma, y pudiendo favorecer la asimilación de los contenidos. Esto afecta positivamente en la nota del alumno y también ayuda al profesor en los controles de asistencia.

Las preguntas del KAHOOT quedan aguardadas, y pueden ser privadas o públicas. Además, pueden servir perfectamente para el siguiente curso académico y ser ampliadas y/o mejoradas.

Sin embargo, uno de los problemas que pueden surgir es que el punto de acceso al que se conecten los alumnos no pueda soportar tanto dispositivo conectado, y haga que la plataforma expulse a algún alumno. Esto es algo que las universidades deben solucionar en los próximos años si se pretende utilizar la gamificación en clase.

Esta tecnología innovadora se encuentra muy arraigada en las universidades estadounidenses y empieza a ser habitual en Europa.

\section{Referencias}

Pintor Holguín, Emilio; Gargantilla Madera, Pedro, Herreros Ruiz-Valdepeñas, Benjamín y López del Hierro, Marta. KAHOOT EN DOCENCIA: UNA ALTERNATIVA PRACTICA A LOS CLICKERS, XI Jornadas Internacionales de Innovación Universitaria: Educar para transformar (2014). Pág. 322-329

Del Cerro Gómez, Gema $\mathrm{M}^{\mathrm{a}}$. APRENDER JUGANDO, RESOLVIENDO: DISEÑANDO EXPERIENCIAS POSITIVAS DE APRENDIZAJE, XII Jornadas Internacionales de Innovación Universitaria, Educar para transformar: Aprendizaje experiencial (2015). Pág. 237-244

Perdue, Diana. INCREASING STUDENT ENGAGEMENT IN MATH WITH ONLINE GAMES AND ELEMENTS OF GAME THEORY, Proceedings for the 43rd Annual Meeting of the Research Council on Mathematics Learning (2016)

(cc) EY-NC-ND 2016, Universitat Politècnica de València 
Marthe Thorine Sunde y Anlaug Gårdsrud Underdal. Investigating QoE in a Cloud-Based Classroom Response System, Norwegian University of Science and Technology (2014) 